\title{
Goethite and Hematite Hybrid Nanosheet Decorated YZnO NRs for Efficient Solar Water Splitting
}

Daniel Commandeur, Joshua McGuckin, Steven Firth, Rong Qian and Qiao Chen*

*Corresponding Author: Qiao.chen@sussex.ac.uk

Department of Chemistry, School of Life Sciences, University of Sussex, Brighton, BN1 9RH, UK

\section{Supplemental Information}

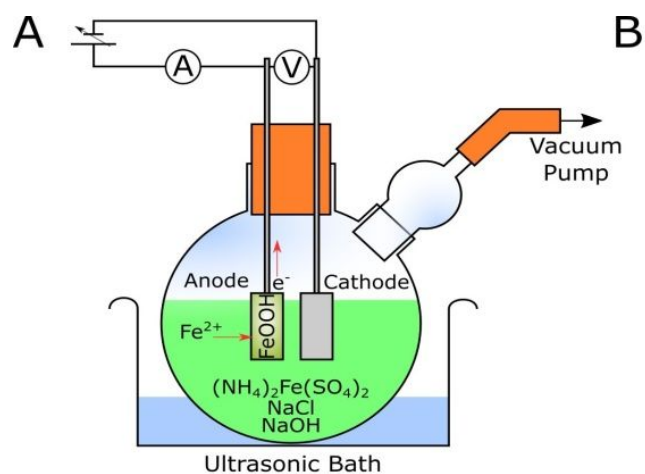

B
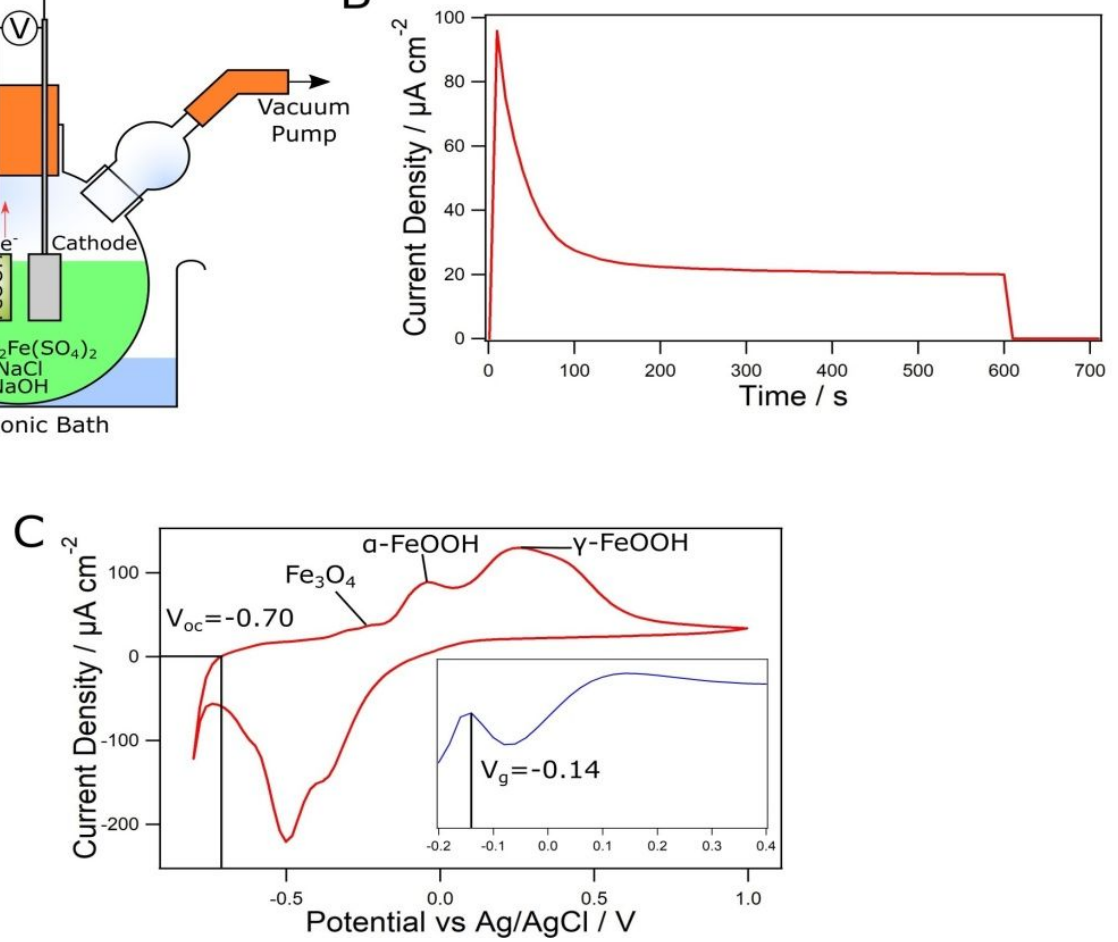

Figure S1: Schematic diagram of the deposition process, A, showing the setup including ultrasonic bath, and electrode positions. B Shows typical deposition current over time, 
integrated to calculate charge density. C Shows the cyclic voltammetry with three electrode setup, used to determine the goethite potential, inset shows a slower scan speed linear sweep for greater accuracy. ${ }^{1}$ Electrode deposition potential was determined by the difference between $\mathrm{V}_{\text {oc }}(-0.70)$ and goethite deposition potential $(-0.14)$ as $0.56 \mathrm{~V}$.
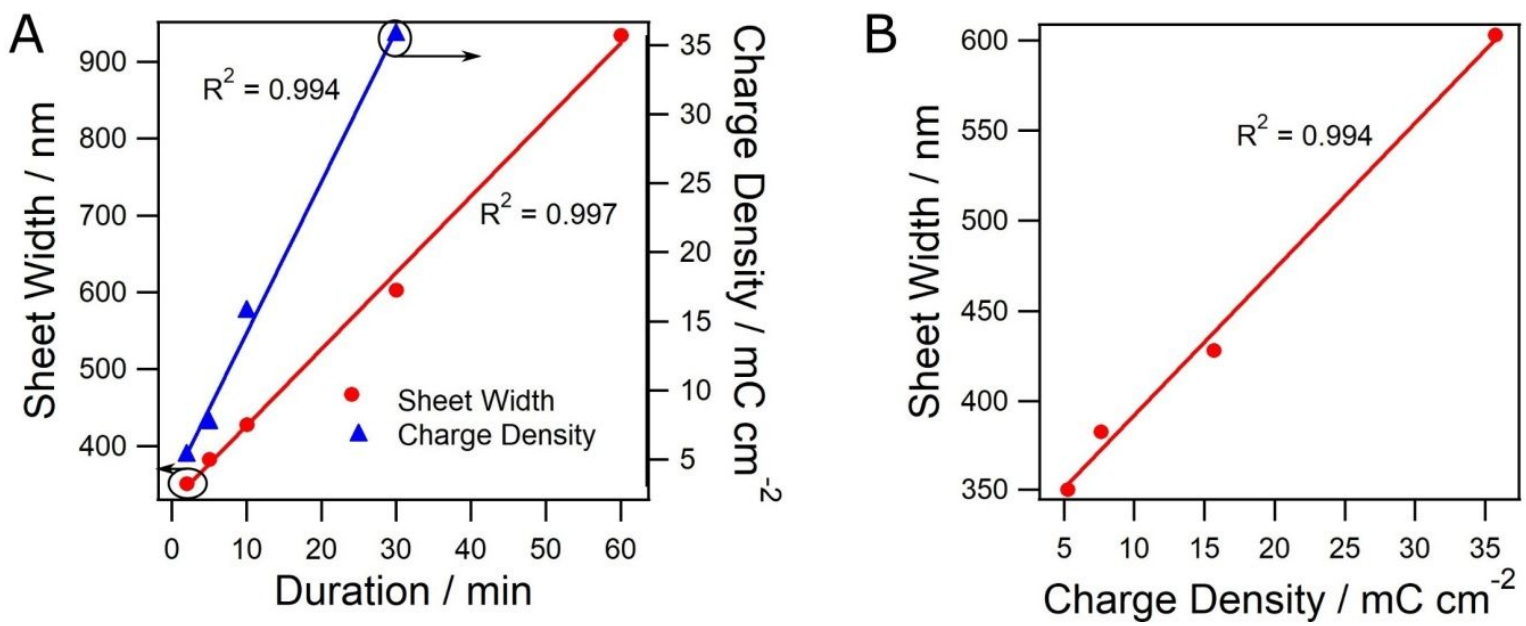

Figure S2: A Shows the integrated total charge density deposited and the nanosheet width against the duration of deposition. B Shows the charge density plotted against nanosheet width. 

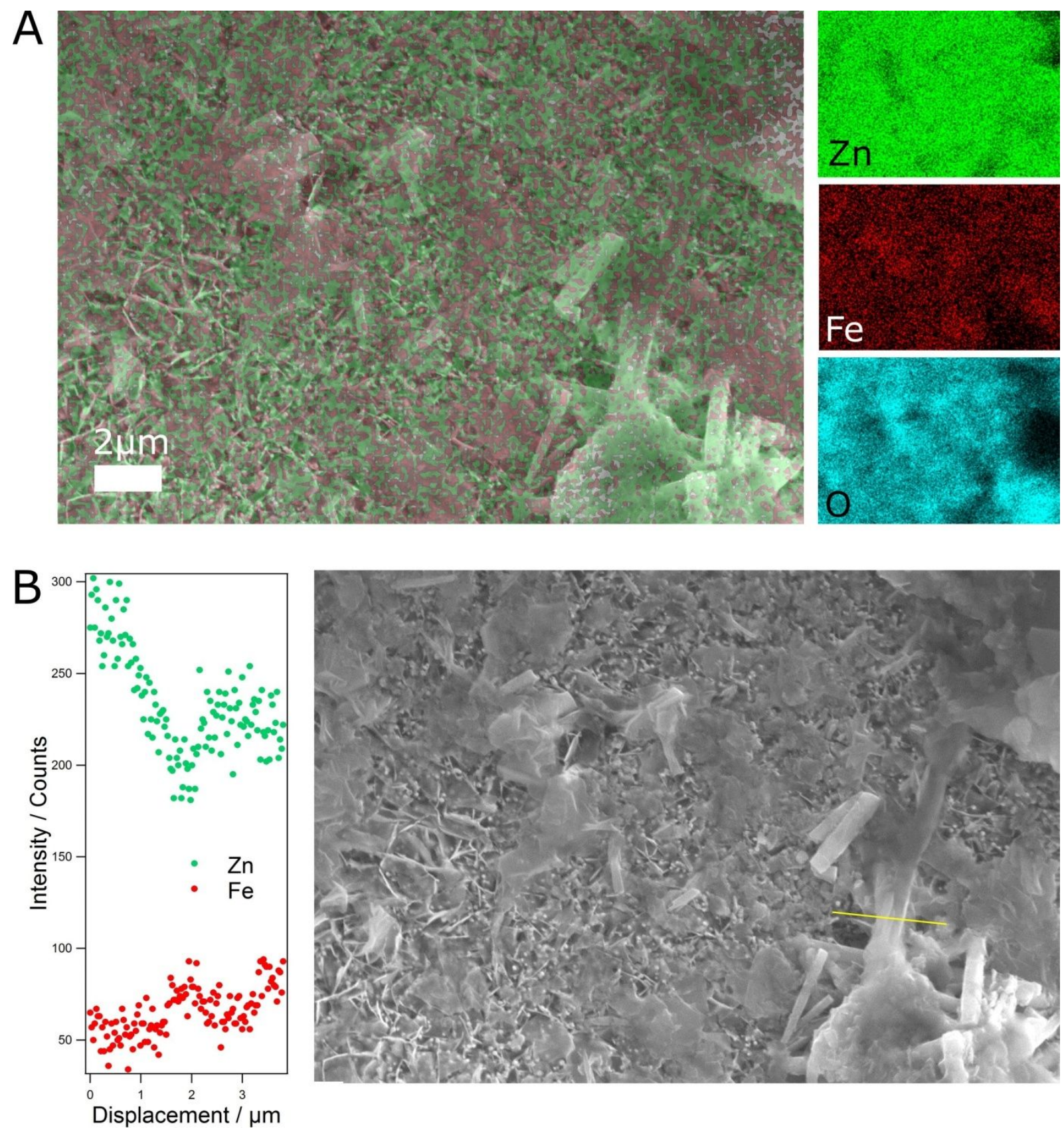

Figure S3: A Shows EDX mapping of the sample area of the 30 min deposition, with composite image of $\mathrm{Zn}$ and $\mathrm{Fe}$ (left) and component elements (right). B Shows an EDX line scan of $\mathrm{Fe}_{2} \mathrm{O}_{3}$ / FeOOH aggregates on the surface. 

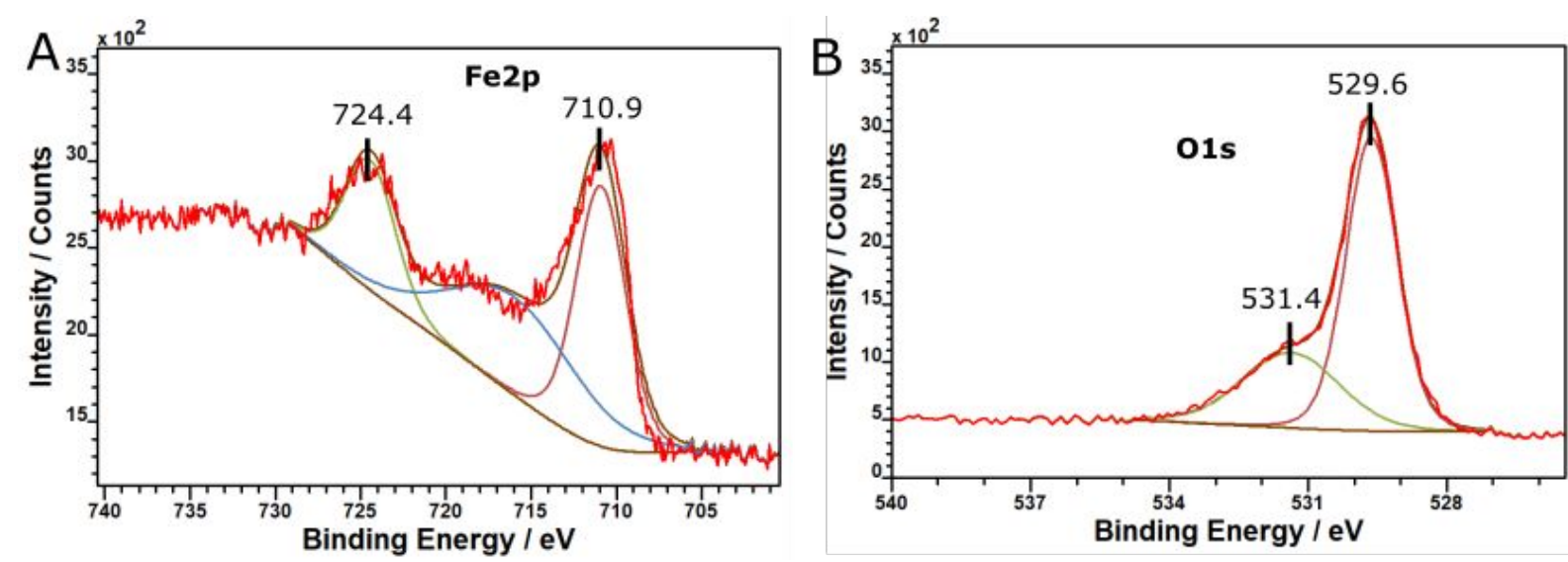

Figure S4: Shows the XPS high resolution scans for $\mathrm{Fe} 2 \mathrm{p}$ and $\mathrm{O} 1 \mathrm{~s}$ in the $\mathrm{Fe}_{2} \mathrm{O}_{3} / \mathrm{FeOOH}$ sample.
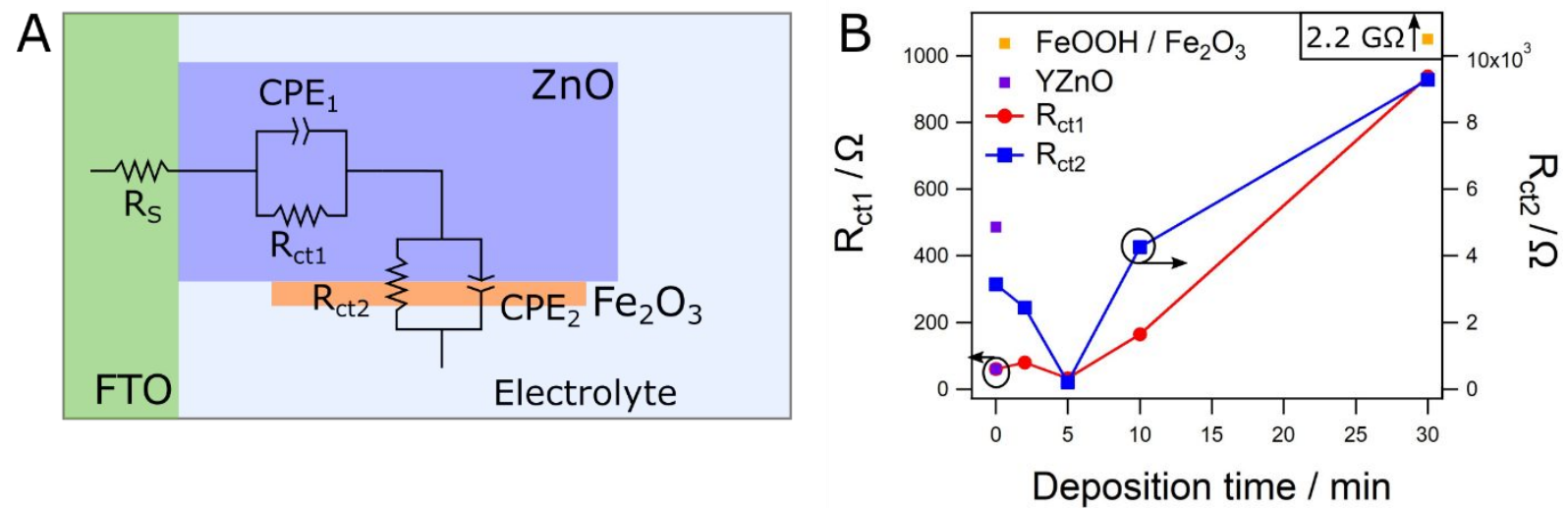

Figure S5: A Shows the equivalent circuit used to fit the Nyquist plots, and B shows the different $\mathrm{R}_{\mathrm{ct}}$ values plotted against deposition duration, along with pure samples. 

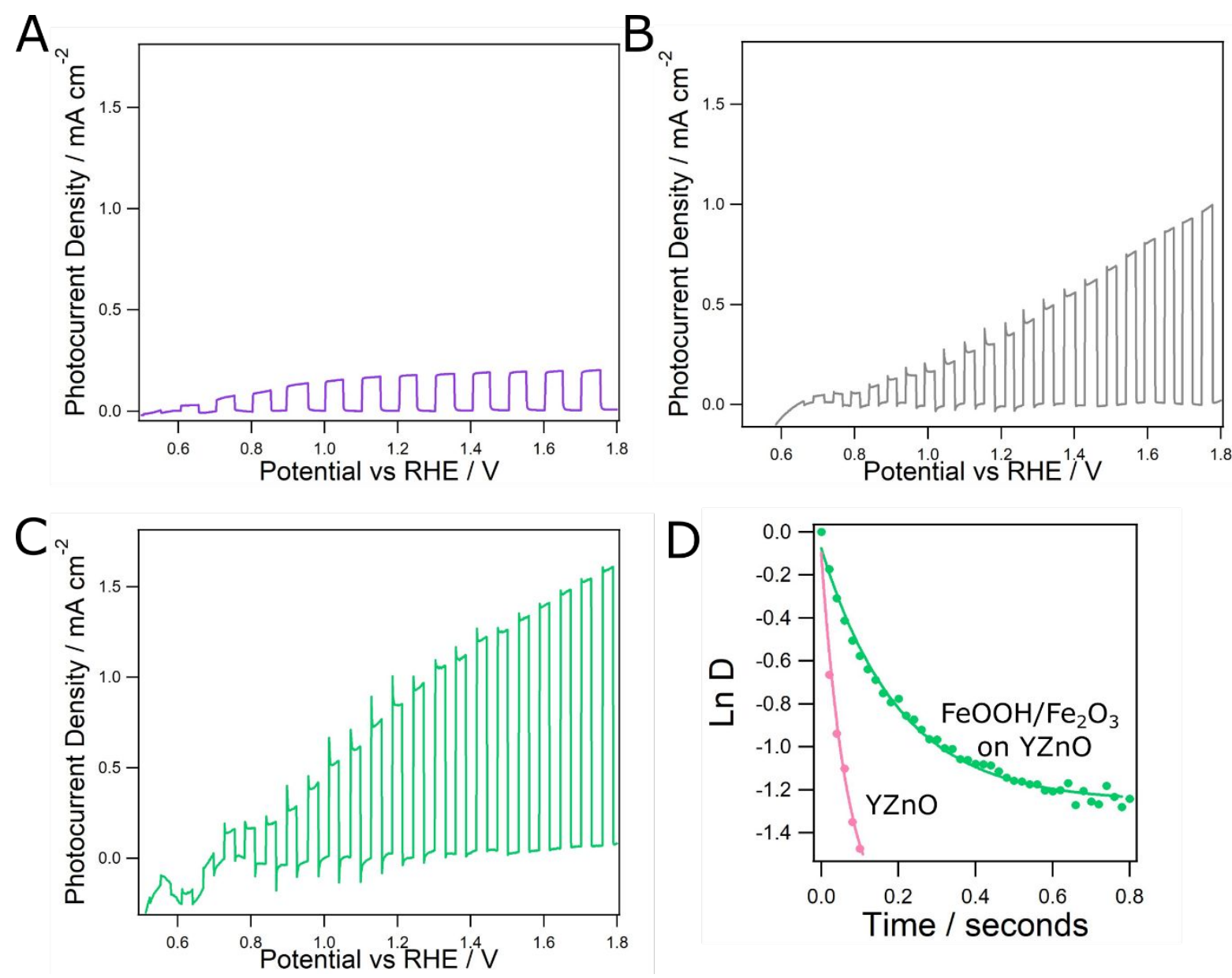

Figure S6: Shows the light/dark stepped linear sweep voltammetry of a comparative selection of anodes, with pristine $\mathrm{ZnO} \mathrm{A}$, and 5 minutes electrodeposition on pure $\mathrm{ZnO} \mathrm{B}$. C Shows the top performing sample, 5 minutes deposition on $\mathrm{Y}$ doped nanowires. Finally $\mathrm{D}$ shows the normalised logarithm of current decay from the chronoamperometry measurements. 


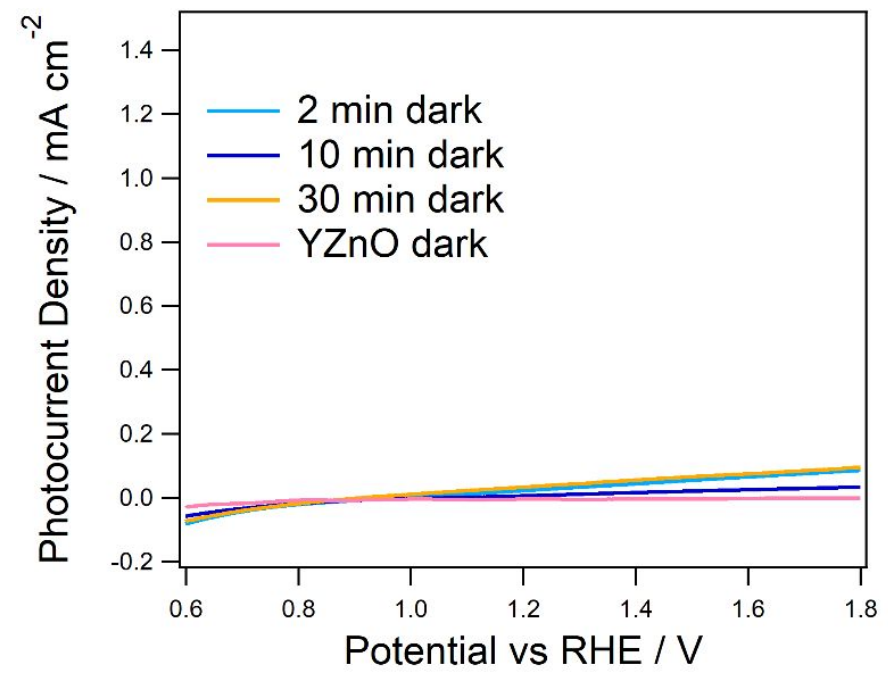

Figure S7: Shows the comparison of all the dark currents for the optimisation.

Table S1: Displays a literature comparison of relevant structures and devices.

\begin{tabular}{|c|c|c|c|}
\hline No. & Sample Description & $\begin{array}{l}\text { Photocurrent Density } \\
\text { at } 1.23 \mathrm{~V}_{\mathrm{RHE}}\end{array}$ & Reference \\
\hline 1 & $\mathrm{NiO}-\mathrm{ZnO}$ on $\mathrm{Fe}_{2} \mathrm{O}_{3}$ films & Negligible & Zhang et al. ${ }^{2}$ \\
\hline 2 & $\mathrm{ZnO}$ quantum dots on $\mathrm{Fe}_{2} \mathrm{O}_{3}$ films & Negligible & Ikram at al. ${ }^{3}$ \\
\hline 3 & $\begin{array}{l}\mathrm{ZnO} \text { NRs covered in } \mathrm{Fe}_{2} \mathrm{O}_{3} \\
\text { nanoparticles }\end{array}$ & Negligible & Chakraborty et al. ${ }^{4}$ \\
\hline 4 & $\begin{array}{l}\mathrm{ZnO} \mathrm{NRs} \text { coated with } \mathrm{Fe}_{2} \mathrm{O}_{3} \text {, prior to } \\
\mathrm{Fe}_{2} \mathrm{PO}_{4} \text { encapsulation }\end{array}$ & $0.85 \mathrm{~mA} \mathrm{~cm}^{-2}$ & Qin et al. ${ }^{5}$ \\
\hline 5 & $\mathrm{ZnO}$ NRs coated with $\mathrm{Fe}_{2} \mathrm{O}_{3}$ & $1.27 \mathrm{~mA} \mathrm{~cm}^{-2}$ & Hsu et al. ${ }^{6}$ \\
\hline 6 & This Work & $0.91 \mathrm{~mA} \mathrm{~cm}^{-2}$ & $\mathrm{n} / \mathrm{a}$ \\
\hline 7 & $\begin{array}{l}\text { Silicon doped nanostructured } \mathrm{Fe}_{2} \mathrm{O}_{3} \\
\text { films }\end{array}$ & $2.2 \mathrm{~mA} \mathrm{~cm}^{-2}$ & Kay et al. ${ }^{7}$ \\
\hline
\end{tabular}


$8 \quad$ Co-doped $\mathrm{ZnO}$ nanorods with a transparent functionalizing MOF

$9 \quad \mathrm{Sn}$ doped $\mathrm{Fe}_{2} \mathrm{O}_{3}$ from FTO

10 Zinc Ferrite modified Al-doped $\mathrm{ZnO}$ NR Arrays
$0.15 \mathrm{~mA} \mathrm{~cm}^{-2}$

$1.0 \mathrm{~mA} \mathrm{~cm}^{-2}$

$1.72 \mathrm{~mA} \mathrm{~cm}^{-2}$
Galan-Gonzalez et $a l .^{8}$

Annamalai et al. ${ }^{9}$

$\mathrm{Xu}$ et al. ${ }^{10}$
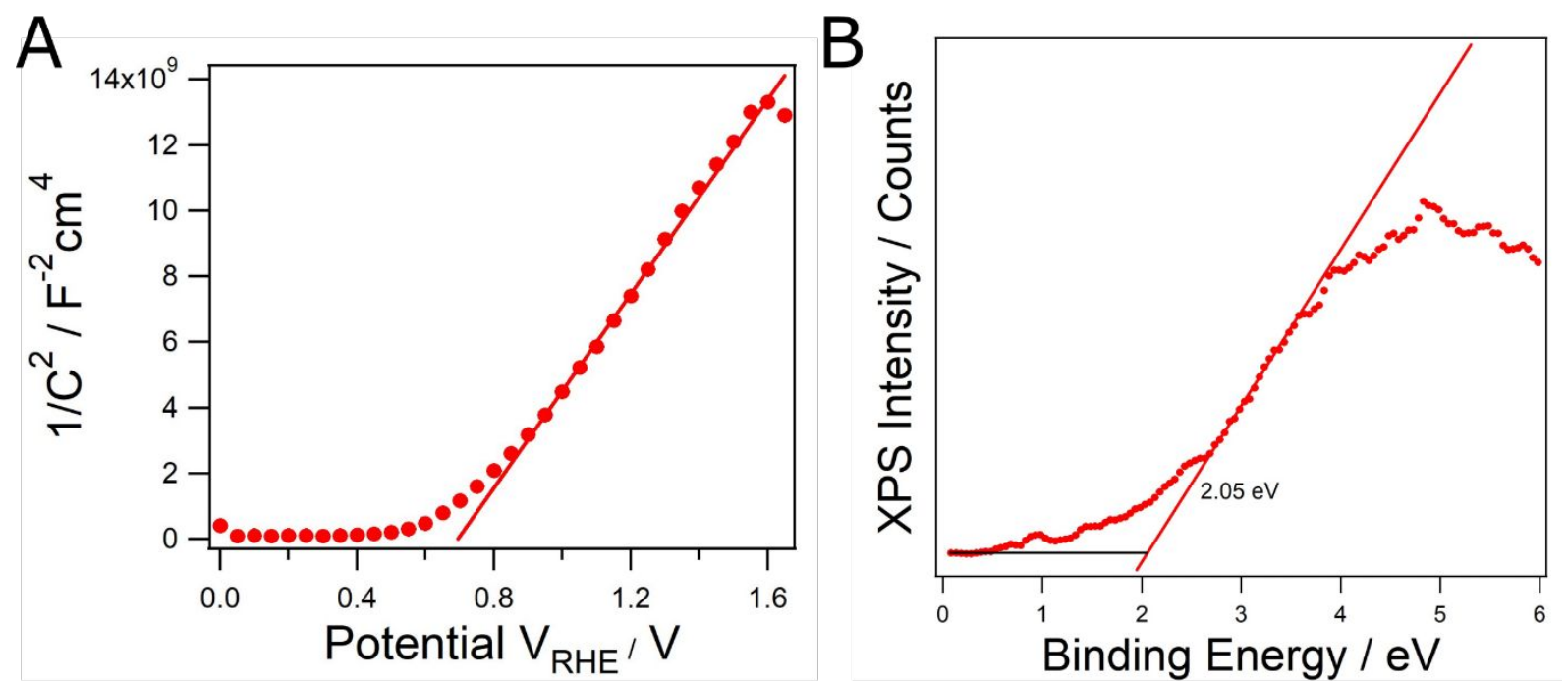

C

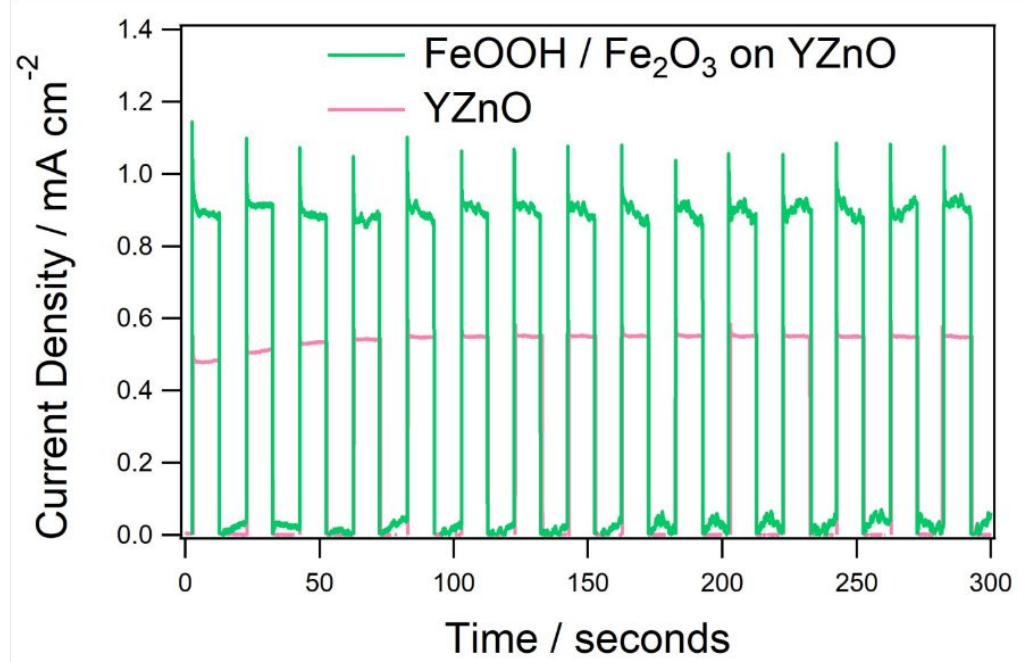

Figure S8: A shows the Mott-Schottky plot of the anodic deposition of $\mathrm{Fe}_{2} \mathrm{O}_{3}$, yielding $\mathrm{V}_{\mathrm{FB}}$ 
value of $0.69 \mathrm{~V}_{\mathrm{RHE}}$ and dopant density of $1.19 \times 10^{26} \mathrm{~m}^{-3}$. B shows the XPS survey scan of the hybrid junction and valence band determination, finally $\mathrm{C}$ shows the chopped illumination chronoamperometry test over a five minute period.

\section{References}

(1) Martinez, L.; Leinen, D.; Martín, F.; Gabas, M.; Ramos-Barrado, J. R.; Quagliata, E.; Dalchiele, E. A. Electrochemical Growth of Diverse Iron Oxide $\left(\mathrm{Fe}_{3} \mathrm{O}_{4}, \alpha-\mathrm{FeOOH}\right.$, and $\gamma$-FeOOH) Thin Films by Electrodeposition Potential Tuning. J. Electrochem. Soc. 2007, 154 (3), D126.

(2) Zhang, C.; Fan, W.; Bai, H.; Yu, X.; Chen, C.; Zhang, R.; Shi, W. SandwichNanostructured NiO-ZnO Nanowires@ $\alpha-\mathrm{Fe}_{2} \mathrm{O}_{3}$ Film Photoanode with a Synergistic Effect and p-n Junction for Efficient Photoelectrochemical Water Splitting. ChemElectroChem 2014, 1 (12), 2089-2097.

(3) Ikram, A.; Sahai, S.; Rai, S.; Dass, S.; Shrivastav, R.; Satsangi, V. R. Enhanced Photoelectrochemical Conversion Performance of $\mathrm{ZnO}$ Quantum Dots Sensitized $\alpha$-Fe 2 O 3 Thin Films. Int. J. Hydrogen Energy 2015, 40 (16), 5583-5592.

(4) Chakraborty, M.; Roy, D.; Biswas, A.; Thangavel, R.; Udayabhanu, G. Structural, Optical and Photo-Electrochemical Properties of Hydrothermally Grown ZnO Nanorods Arrays Covered with $\alpha-\mathrm{Fe}_{2} \mathrm{O}_{3}$ Nanoparticles. RSC Adv. 2016, 6 (79), 75063-75072.

(5) Qin, D. D.; Tao, C. L. A Nanostructured $\mathrm{ZnO}-\mathrm{ZnFe}_{2} \mathrm{O}_{4}$ Heterojunction for the Visible Light Photoelectrochemical Oxidation of Water. RSC Adv. 2014, 4 (33), 16968.

(6) Hsu, Y. K.; Chen, Y. C.; Lin, Y. G. Novel $\mathrm{ZnO} / \mathrm{Fe}_{2} \mathrm{O}_{3}$ Core-Shell Nanowires for Photoelectrochemical Water Splitting. ACS Appl. Mater. Interfaces 2015, 7 (25), 1415714162.

(7) Kay, A.; Cesar, I.; Grätzel, M. New Benchmark for Water Photooxidation by Nanostructured $\alpha-\mathrm{Fe}_{2} \mathrm{O}_{3}$ Films. J. Am. Chem. Soc. 2006, 128 (49), 15714-15721.

(8) Galán-González, A.; Sivan, A. K.; Hernández-Ferrer, J.; Bowen, L.; Di Mario, L.; Martelli, F.; Benito, A. M.; Maser, W. K.; Chaudhry, M. U.; Gallant, A.; et al. CobaltDoped ZnO Nanorods Coated with Nanoscale Metal-Organic Framework Shells for Water-Splitting Photoanodes. ACS Appl. Nano Mater. 2020, 3 (8), 7781-7788.

(9) Annamalai, A.; Subramanian, A.; Kang, U.; Park, H.; Choi, S. H.; Jang, J. S. Activation of Hematite Photoanodes for Solar Water Splitting: Effect of FTO Deformation. J. Phys. Chem. C 2015, 119 (7), 3810-3817.

(10) Xu, Y. F.; Rao, H. S.; Wang, X. D.; Chen, H. Y.; Kuang, D. Bin; Su, C. In Situ Formation of Zinc Ferrite Modified Al-Doped ZnO Nanowire Arrays for Solar Water Splitting. $J$. Mater. Chem. A 2016, 4 (14), 5124-5129. 